\title{
Two-Dimensional Nutation NQR Broad-Line Spectra in Oriented Samples
}

\author{
Nicolay Sinyavsky ${ }^{\mathrm{a}}$, Irina Korneva ${ }^{\mathrm{a}}$, Michał Ostafin ${ }^{\mathrm{b}}$, Bolesław Nogaj ${ }^{\mathrm{b}}$, and \\ Mariusz Maćkowiak ${ }^{\mathrm{c}}$ \\ ${ }^{a}$ Baltic State Academy, Molodiozhnaya str. 6, 236029, Kaliningrad, Russia \\ b Department of Physics, Adam Mickiewicz University, Umultowska 85, 61-614, Poznań, Poland \\ c Institute of Molecular Physics, Polish Academy of Sciences, ul. Smoluchowskiego 17, 60-179, \\ Poznań, Poland \\ Reprint requests to Prof. M. M.; Fax: 48-61-8684-524; E-mail: mackow@ifmpan.poznan.pl
}

Z. Naturforsch. 61a, 499 - 504 (2006); received March 22, 2006

The NQR nutation method to determine the electric field gradient asymmetry parameter $\eta$ in systems, where the resonance line is so broad that the radio frequency field can excite only a portion of the nuclear spins, is presented. In this situation, the recently developed spectroscopic methods are not applicable. Two-dimensional nutation NQR spectra of oriented powders are calculated and used to determine $\eta$ at particular frequencies along a broad NQR line. The proposed method is useful for single crystals, oriented powders, glasses, and disordered systems even for small values of the asymmetry parameter. Therefore it can be used to evaluate fluctuations in $\eta$ and the quadrupole coupling constant $e^{2} q Q$ due to inhomogeneities. We demonstrate the application of this method to oriented chalcogenide semiconducting glasses.

Key words: Nuclear Quadrupole Resonance; 2D Nutation Spectroscopy; Electric Field Gradient Tensor.

\section{Introduction}

The nuclear quadrupole coupling constant $e^{2} q Q$ and the electric gradient tensor asymmetry parameter $\eta$ are extremely sensitive to the electron distribution around the quadrupolar nucleus. Pure nuclear quadrupole resonance (NQR) is a very useful tool for studying the molecular structure and electronic properties of materials without the need to apply an external magnetic field. The existence of a single quantization axis as determined by the external magnetic field - leads in NMR spectroscopy to a dependence of an NMR spectrum on the relative orientations of the Zeeman and perturbing second-rank tensor interactions. Such an axis is absent in zero-field NQR spectroscopy. For the $\operatorname{spin} I=3 / 2$ it is not possible to determine the second-rank quadrupolar tensor from a conventional one-dimensional zero-field NQR, since the transition frequency depends on both the quadrupolar coupling constant $e^{2} q Q$ and the asymmetry parameter $\eta$. In recent years, some experimental methods have been invented to extract these parameters without applying a magnetic field. This includes 2D nutation spectroscopy $[1,2]$. This method uses two time periods. The first time period, characterized by a time variable $t_{1}$, is the duration of the radiofrequency excitation pulse. The second period is characterized by a time variable $t_{2}$ and is the period of free precession of a quadrupolar nucleus at zero field. However, this method is based on the assumptions that the NQR line is much narrower than the effective nutation frequency [which is of the order of $\gamma B_{1}$, where $B_{1}$ is the radio frequency (RF) magnetization and $\gamma$ is the nuclear gyromagnetic ratio]. In practice, there are many systems where these requirements are not met; superconductors, magnetic materials, and systems with disorder, like glasses, are just a few examples. 2D nutation NQR spectroscopy can be directly applied neither to broad powder lines nor to inhomogeneously broaden single crystal lines of disordered systems like glasses or incommensurate systems [3]. The inhomogeneous broadening is normally a consequence of the lattice disorder, like topological disorder in glasses or the presence of a large amount of impurities in substitutionally disordered systems. In all cases, the EFG tensors at the sites of resonating nuclei vary with space. A single sharp resonance frequency $\omega_{0}$ is replaced by a frequencydistribution function $f\left(\omega_{0}\right)$, which is characteristic for various disorder types. The inhomogeneous frequencydistribution function $f\left(\omega_{0}\right)$ determines the inhomoge- 

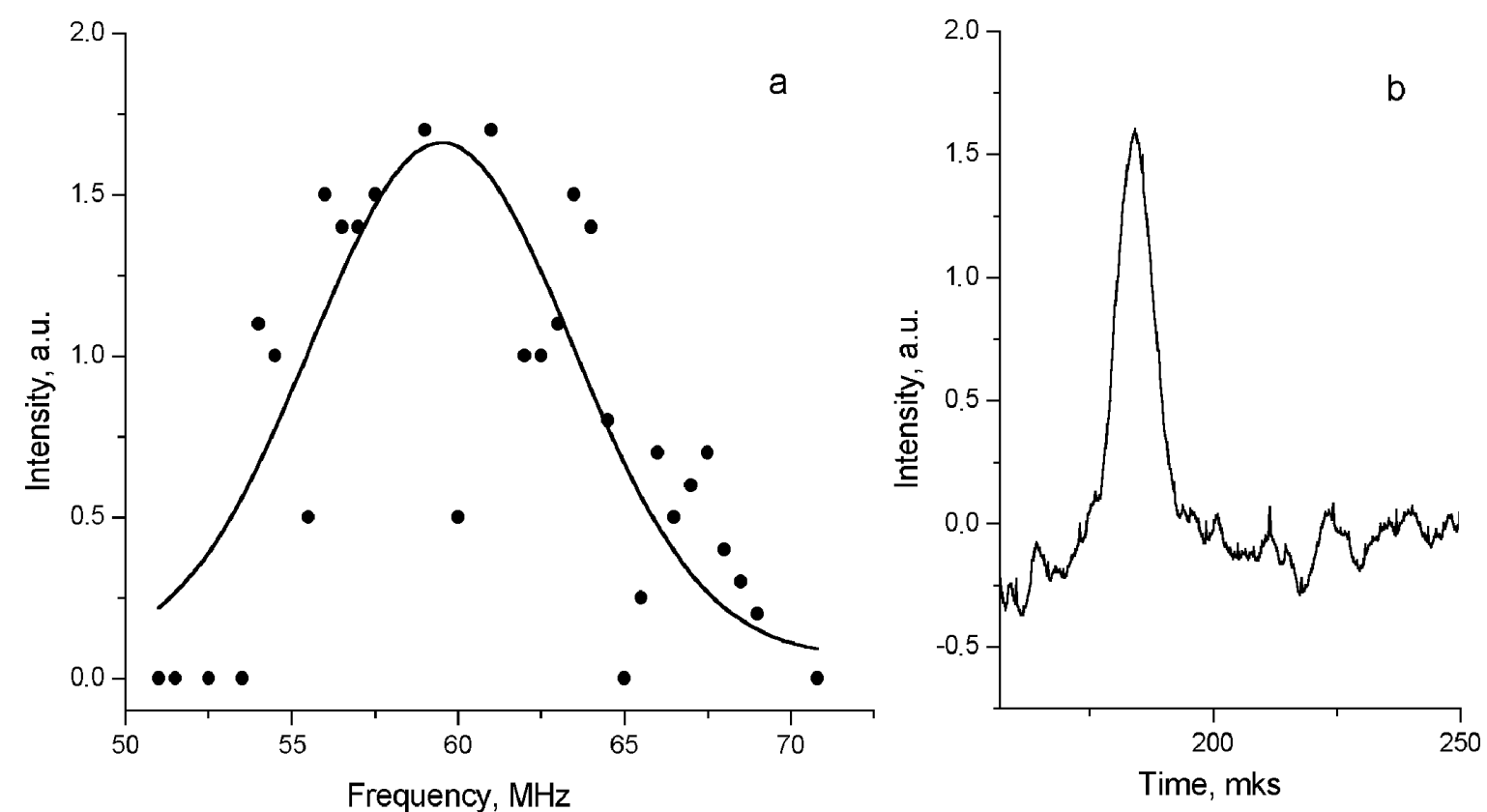

Fig. 1. (a) NQR spectrum of ${ }^{75} \mathrm{As}$ and (b) the spin-echo signal in $\mathrm{As}\left(\mathrm{Se}_{0.88} \mathrm{Te}_{0.12}\right)_{1.5}$ at $77 \mathrm{~K}$.

neous NQR lineshape. In broad-line solids the nutation frequency changes with the irradiation offset. The nutation lineshape varies over the NQR spectrum in a complicated way. The rather simple nutation lineshapes, which can be used for the asymmetry parameter determination, are obtained only in the onresonance irradiated parts of the NQR spectrum. Even there, the presence of finite homogeneous linewidth smears and obscures the characteristic shapes of the nutation lines. For broad NQR lines the nutation spectrum exhibits a characteristic "banana-like" shape due to irradiation offset effects [3].

In chalcogenide semiconducting glasses the NQR lineshape is very sensitive to the fluctuations of the order parameter, thus providing a measure of the system's homogeneity. However, the conventional onedimensional NQR spectroscopy of ${ }^{75}$ As nuclei is not informative due to the very broad line. In disordered systems one is primarily interested in studying the distribution of $e^{2} q Q$ and $\eta$ values around some mean value, reflecting the local deviations of the average lattice-site symmetry. The main idea of the new method, proposed in this work, is to measure the 2D nutation NQR spectra for selectively excited spin-echo signals in powder samples preliminarily oriented in a strong magnetic field. The method can be applied for single crystals, oriented powders or glasses, where the orientation of the EFG tensor acting on all studied nuclei is characterized by the same value of the polar angle $\theta$. The experimental configuration is strictly determined. In order to get oriented samples, the powder is mixed with a liquid epoxy resin, placed in a strong magnetic field of several $\mathrm{T}$ and hardened by drying or quenching. The preliminarily oriented sample is placed in a coil so that the symmetry axis of the coil is pointing in the direction perpendicular to the $z$ axis of the EFG tensor $(\theta=\pi / 2)$.

\section{Results and Discussion}

The experimental ${ }^{75} \mathrm{As}$ NQR spectrum of a chalcogenide semiconducting glass, $\mathrm{As}\left(\mathrm{Se}_{0.88} \mathrm{Te}_{0.12}\right)_{1.5}$ (disordered powder), at $77 \mathrm{~K}$ is shown in Figure 1a. The NQR line is measured with a home-built pulse spectrometer to which an automated frequency sweep feature is added. The circuit remains tuned and matched throughout the frequency sweep. The NQR line is centered at about $59 \mathrm{MHz}$ and has a $10 \mathrm{MHz}$ width. The NQR spin-echo signal taken at $58.5 \mathrm{MHz}$ is displayed in Figure 1b. The spin-echo signal has been obtained with a two-pulse sequence for a first-pulse length of $4 \mu \mathrm{s}$; the pulse-interval was $70 \mu \mathrm{s}$; the repetition time $100 \mathrm{~ms}$, the dwell-time $0.2 \mu \mathrm{s}$, the number of scans 4000, and the filter band-width $100 \mathrm{kHz}$. 
The sample was kept in liquid nitrogen, which prevented a temperature drift of the NQR frequency. The amplitude of the exciting RF field in the frequency units $\omega_{\mathrm{r}}$ is much smaller than the NQR line-width $\Delta \omega_{0}\left(\omega_{\mathrm{r}} \ll \Delta \omega_{0}\right)$. Thus only a portion of the spectrum is excited and the main contribution to the observed spin-echo signal is given by nuclear spins with the resonance frequency close to the exciting pulse frequency $\omega$. The magnetization vectors of corresponding isochromats with the frequency within the range $\left|\omega_{0}-\omega\right| \leq \omega_{\mathrm{r}}$ are rotating almost by the same angle.
Thus the spin-echo signal width is of the order $\sim \pi / \omega_{\mathrm{r}}+t_{\mathrm{w}}$ (Fig. 1b). In our experiment $\omega_{\mathrm{r}}=\gamma B_{1}=$ $2 \pi \cdot 140 \mathrm{kHz}$, and this value has been assumed for computer simulations.

For nuclei with $I=3 / 2$ the spin-echo intensity, obtained by a two-pulse sequence, can be calculated using the procedure described in [4]. In the cited reference, the response of a system of spins $I=3 / 2$ in a zero-applied field, experiencing electric quadrupole couplings, to the two-pulse sequence is analyzed. The spin-echo intensity following the second pulse is given [5] by

$$
I\left(t_{1}, t_{2}\right)=\frac{m^{2}}{8 \alpha \xi^{2}}\left\{\begin{array}{c}
\cos \Delta \omega t_{2}\left[\begin{array}{c}
\frac{\Delta \omega}{\xi}\left(A \sin \Delta \omega t_{1}-\frac{\Delta \omega}{2 \xi} \sin 2 \xi t_{\mathrm{w}}^{\prime} \cos \Delta \omega t_{1}\right) \\
+\left(1-\frac{\Delta \omega}{2 \xi}\right)\left(A \sin (2 \xi+\Delta \omega) t_{1}-\frac{\Delta \omega}{2 \xi} \sin 2 \xi t_{\mathrm{w}}^{\prime} \cos (2 \xi+\Delta \omega) t_{1}\right) \\
+\left(1-\frac{\Delta \omega}{2 \xi}\right)\left(A \sin (2 \xi-\Delta \omega) t_{1}+\frac{\Delta \omega}{2 \xi} \sin 2 \xi t_{\mathrm{w}}^{\prime} \cos (2 \xi-\Delta \omega) t_{1}\right)
\end{array}\right]+ \\
\sin \Delta \omega t_{2}\left[\begin{array}{c}
\frac{\Delta \omega}{\xi}\left(A \cos \Delta \omega t_{1}-\frac{\Delta \omega}{2 \xi} \sin 2 \xi t_{\mathrm{w}}^{\prime} \sin \Delta \omega t_{1}\right) \\
+\left(1-\frac{\Delta \omega}{2 \xi}\right)\left(A \cos (2 \xi+\Delta \omega) t_{1}-\frac{\Delta \omega}{2 \xi} \sin 2 \xi t_{\mathrm{w}}^{\prime} \sin (2 \xi+\Delta \omega) t_{1}\right) \\
+\left(1-\frac{\Delta \omega}{2 \xi}\right)\left(-A \cos (2 \xi-\Delta \omega) t_{1}+\frac{\Delta \omega}{2 \xi} \sin 2 \xi t_{\mathrm{w}}^{\prime} \sin (2 \xi-\Delta \omega) t_{1}\right.
\end{array}\right)
\end{array}\right\},
$$

where $A=\left(\cos ^{2} \xi t_{\mathrm{w}}^{\prime}-\frac{\Delta \omega^{2}}{4 \xi^{2}} \sin ^{2} \xi t_{\mathrm{w}}^{\prime}\right)$, and other variables are defined by $\alpha=\frac{\gamma B_{1}}{4}, \xi=\frac{1}{2} \sqrt{4 m^{2}+\Delta \omega^{2}}$, $m=\frac{\alpha R(\theta, \varphi)}{\sqrt{3+\eta^{2}}}$.

The orientation function $R(\theta, \varphi)$ depends on the polar and azimuthal angles and $\varphi$ of the RF coil direction in the EFG tensor eigenframe and asymmetry parameter $\eta$ as

$$
R(\theta, \varphi)=\sqrt{4 \eta^{2} \cos ^{2} \theta+\sin ^{2} \theta\left(9+\eta^{2}+6 \eta \cos 2 \varphi\right)} .
$$

Here $t_{1}=t_{\mathrm{w}}$ is the length of the first (nutation) pulse, $t_{\mathrm{w}}^{\prime}$ the length of the second (refocusing) pulse, $t_{2}$ the time variable calculated from the top of the echo-signal, and $\Delta \omega=\omega-\omega_{0}$ the frequency offset $\left(\omega_{2}=\Delta \omega\right)$.

In (1) the length of the refocusing pulse is taken into account, contrary to [3]. As follows from (1), the 2D nutation spectrum will consist of the following frequencies:

$$
\omega_{1}=\left\{\begin{array}{l}
2 \xi+\omega_{2}, \\
\omega_{2} .
\end{array}\right.
$$

In oriented powders the $z$ direction is well defined, but the $x y$ directions are interchangeable. The samples are usually made from grains of single crystals oriented in a magnetic field. However, the $x$ and $y$ directions are random. Thus the averaging can be done only over the $\varphi$ variable.

The theoretical 2D NQR nutation spectra in oriented powders are shown in Figs. 2 and 3. The spectra have been calculated assuming a Gaussian shape of the frequency-distribution function $f\left(\omega_{0}\right)$ with $\sigma=2 \pi \cdot 60 \mathrm{kHz}$ and the integration interval $-3 \sigma \leq \Delta \omega \leq 3 \sigma$ [3]. The other parameters used were: $T_{2}=100 \mu \mathrm{s}, \tau=200 \mu \mathrm{s}$ (a phenomenological damping constant for the nutation domain $\omega_{1}$ ), $t_{\mathrm{w}}^{\prime}=1 \mu \mathrm{s}, \omega_{\mathrm{r}}=\gamma B_{1}=2 \pi \cdot 140 \mathrm{kHz}$, the incrementation steps $\Delta t_{1}=1 \mu \mathrm{s}, \Delta t_{2}=2 \mu \mathrm{s}$. The refocusing pulse affects the singularities in the nutation spectrum. The spectrum becomes distorted and the distortion changes with the length of the refocusing pulse. The positions of singularities, however, remain unchanged. For short refocusing pulses $\left(t_{\mathrm{w}}^{\prime} \approx 1 \mu \mathrm{s}\right)$ the distortions are very small. This effect is illustrated in 

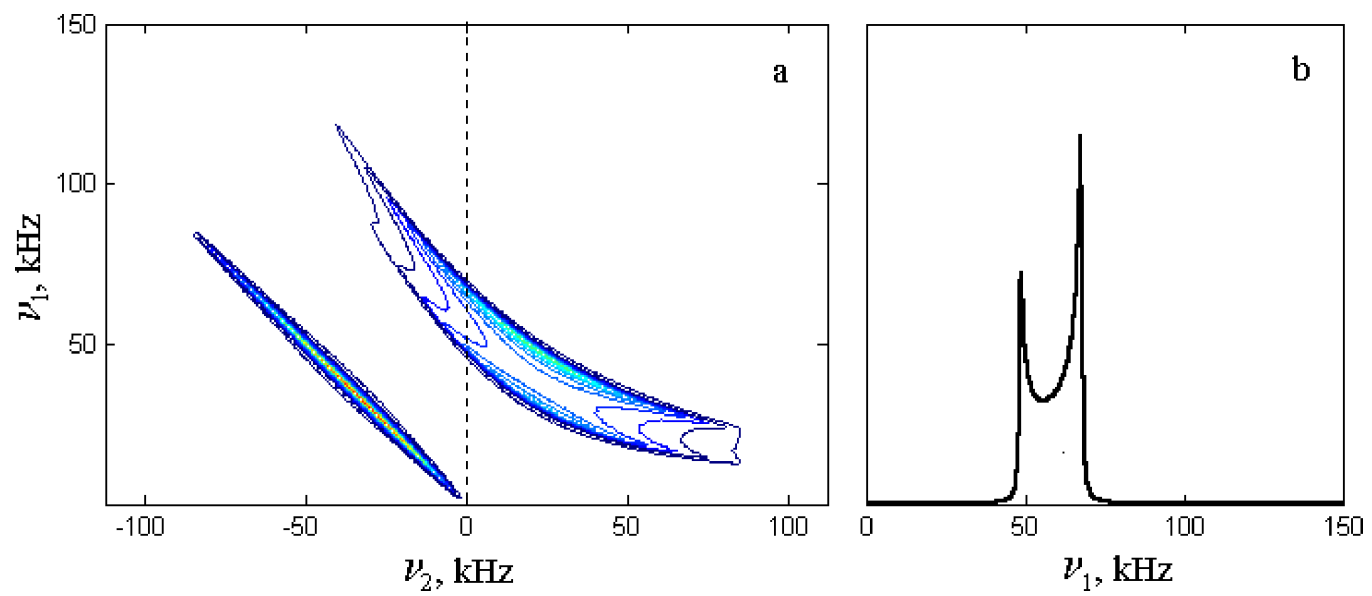

Fig. 2. (a) Two-dimensional NQR nutation spectrum $(I=3 / 2)$ in oriented powder for $\eta=0.5, \theta=\pi / 2$ and (b) corresponding one-dimensional nutation spectrum sectioned at $v_{2}=0$.
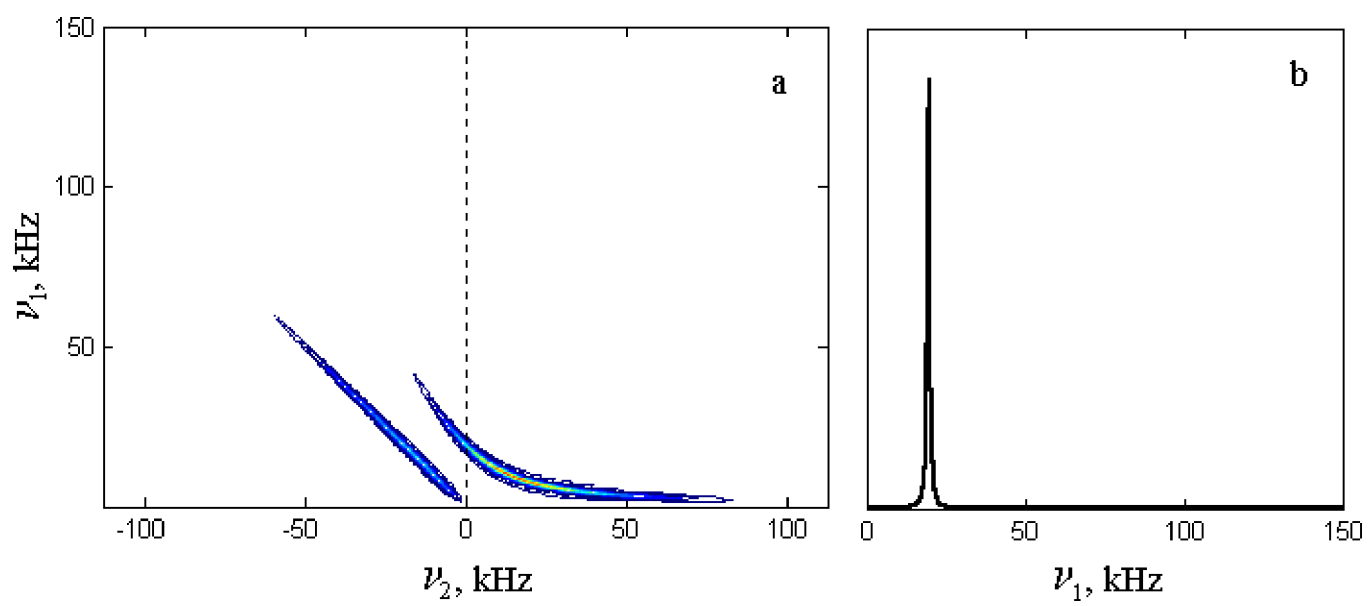

Fig. 3. The same spectrum as in Fig. 2, but for a different position of the oriented sample $(\theta=0)$.
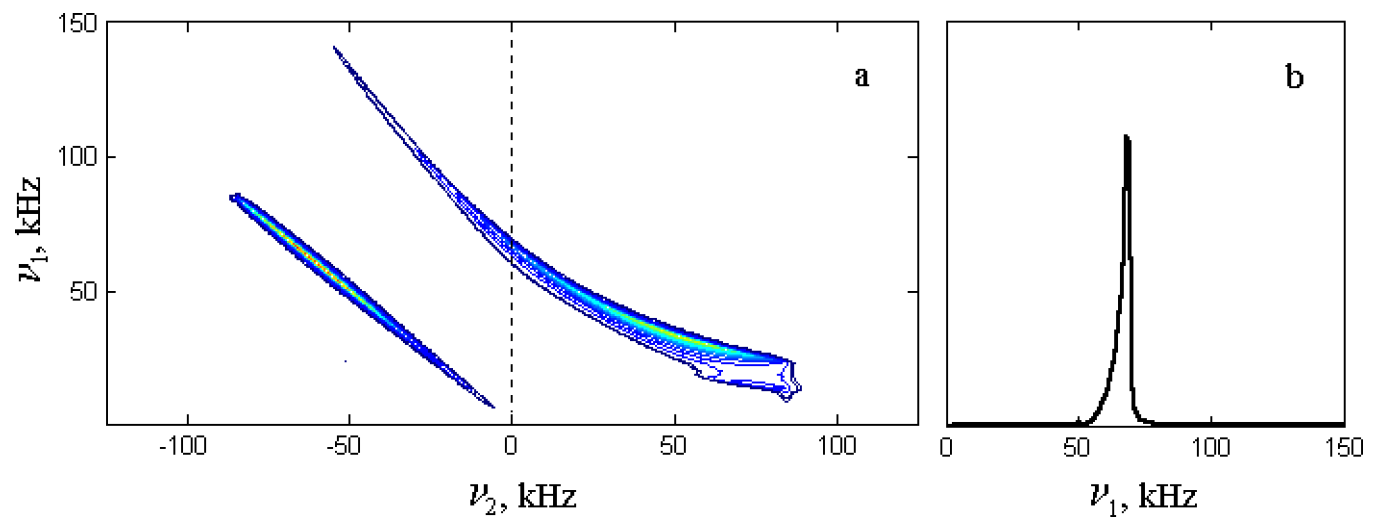

Fig. 4. (a) Two-dimensional NQR nutation spectrum $(I=3 / 2)$ in oriented powder for $\eta=0.5, \theta=\pi / 2$ and (b) corresponding one-dimensional nutation spectrum sectioned at $v_{2}=0$ for the length $t_{\mathrm{w}}^{\prime}=5 \mu \mathrm{s}$ of the refocusing pulse. 

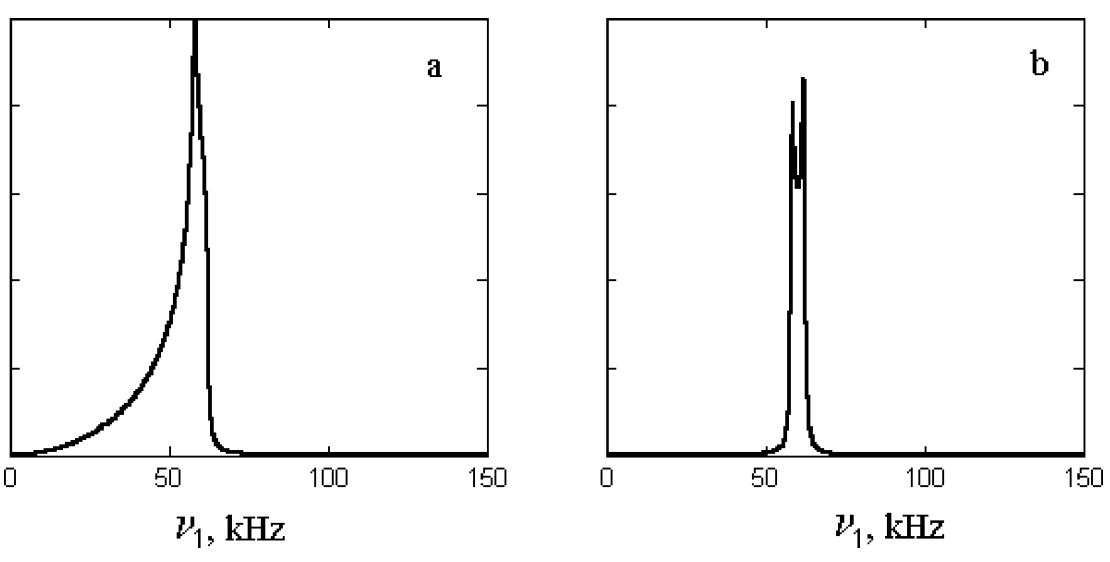

Fig. 5. One-dimensional nutation NQR spectrum sectioned at $v_{2}=0$ for $\eta=0.1$ in (a) isotropic powder and (b) oriented powder.
Figs. 2 and 4, where the refocusing pulse changes from $1 \mu$ s to $5 \mu \mathrm{s}$.

The positions of singularities in the nutation spectra of ordered samples and powders are the same. However, the lineshapes of the nutation spectra are different. For the ordered sample, the function describing the nutation spectra intensities for $\theta=\pi / 2$ and $\Delta \omega=0$ may be calculated and written as

$$
I(\omega)=\frac{2}{\pi} \frac{\omega}{\sqrt{b^{2}-(\omega-a)^{2}}},
$$

where $a=\frac{9+\eta^{2}}{4\left(3+\eta^{2}\right)}, b=\frac{6 \eta}{4\left(3+\eta^{2}\right)}$ and the nutation frequency $\omega$ is normalized to $\omega_{\mathrm{r}}=\gamma B_{1}$.

The normalized frequencies of the nutation singularities are the same as for powders, namely $\omega_{\mathrm{n} 1}=\frac{\eta}{\sqrt{3+\eta^{2}}}$ and $\omega_{\mathrm{n} 2,3}=\frac{3 \mu \eta}{2 \sqrt{3+\eta^{2}}}$. For the case $\theta=0, \Delta \omega=0$ the nutation line is very narrow, as seen in Figure 3. For the intermediate angles $\theta<90^{\circ}$ the resolution of the nutation spectrum in ordered samples is lower. The positions of singularities depend on the value of $\theta$. The difference between the frequencies $\omega_{\mathrm{n} 3}$ and $\omega_{\mathrm{n} 2}$ of the singularities is given by

$\omega_{\mathrm{n} 2, \mathrm{n} 3}=\frac{\gamma B_{1}}{2 \sqrt{3+\eta^{2}}} \sqrt{4 \eta^{2} \cos ^{2} \theta+\sin ^{2} \theta\left(3 \mu \eta^{2}\right)}$.

As shown in Figs. 2, 3, and 4 the diagonal component of the simulated spectra at the frequency $\omega_{1}=-\omega_{2}$ does not provide any information on the EFG asymmetry parameter. The second component ("banana-like" shape) exhibits singularities determined by $\eta$ (for $\theta=\pi / 2$ ) :

$$
\omega_{1}=f\left(\omega_{2}\right)=\sqrt{\omega_{2}^{2}+\frac{\left(\gamma B_{1}\right)^{2}}{4\left(3+\eta^{2}\right)}(3 \pm \eta)^{2} \pm \omega_{2}} .
$$

From the practical point of view the most interesting feature is, that for ordered powders the nutation singularities have the form of very sharp peaks. In ordinary isotropic powders the nutation spectra singularities show broad shoulders, thus giving rise to difficulties in accurate spectral analysis and precise determination of singularities in a continuum-like powder pattern (Fig. 5). In ordered powders the singularities $v_{\mathrm{n} 2}$ and $v_{\mathrm{n} 3}$ may be determined with high accuracy even for small values of $\eta$ (Fig. 5b). The value of the asymmetry parameter $\eta$ can be evaluated directly from the 2D nutation spectra sectioned at $v_{2}=0$ by using the formula

$$
\eta=\frac{3\left(v_{\mathrm{n} 3}-v_{\mathrm{n} 2}\right)}{v_{\mathrm{n} 3}+v_{\mathrm{n} 2}} .
$$

In [5] the angle-dependent NQR method to determine the EFG asymmetry parameter $\eta$ along a broad NQR line in oriented powders was proposed. This method is based on the variation of the spin-echo intensity as a function of $\theta$ in oriented powders and requires the rotation of the sample inside a resonance coil. However, this leads to many artifacts and low accuracy of the data. Our nutation method is free of these limitations, provides high accuracy and does not require the rotation of the sample.

\section{Concluding Remarks}

We have demonstrated that $2 \mathrm{D}$ nutation spin-echo NQR spectroscopy could be used to determine $\eta$ and the EFG tensor at particular frequencies along a broad NQR line in oriented powders. This high-accuracy method can be used to evaluate fluctuations in $\eta$ and the quadrupole coupling constant $e^{2} q Q$ due to inhomogeneities. 
Acknowledgement

One of us (N. S.) thanks the Russian Foundation for Basic Research (RFBR, project no. 05-03-33264) for financial support.

[1] G. S. Harbison, A. Slokenbergs, and T.M. Barbara, J. Chem. Phys. 90, 5292 (1989).

[2] G. S. Harbison and A. Slokenbergs, Z. Naturforsch. 45a, 575 (1990)

[3] J. Dolinsek, F. Milia, G. Papavassiliou, G. Papantopoulos, and R. Rumm, J. Magn. Reson. A 114, 147 (1995).
[4] S. Levy and A. Keren, J. Magn. Reson. 167, 317 (2004).

[5] N. Sinyavsky, N. Velikite, and M. Maćkowiak, Mol. Phys. 99, 1653 (2001). 